\title{
CAPTURE-TIME EXTREMAL COP-WIN GRAPHS
}

\author{
DAVID OFFner \\ Department of Mathematics and Computer Science \\ Westminster College \\ New Wilmington, PA, U.S.A. \\ e-mail: offnerde@westminster.edu \\ AND \\ KERRY OJAKIAN \\ Department of Mathematics and Computer Science \\ Bronx Community College (CUNY) \\ Bronx, NY, U.S.A \\ e-mail: kerry.ojakian@bcc.cuny.edu
}

\begin{abstract}
We investigate extremal graphs related to the game of Cops and Robbers. We focus on graphs where a single cop can catch the robber; such graphs are called cop-win. The capture time of a cop-win graph is the minimum number of moves the cop needs to capture the robber. We consider graphs that are extremal with respect to capture time, i.e., their capture time is as large as possible given their order. We give a new characterization of the set of extremal graphs. For our alternative approach we assign a rank to each vertex of a graph, and then study which configurations of ranks are possible. We partially determine which configurations are possible, enough to prove some further extremal results. We leave a full classification as an open question.
\end{abstract}

Keywords: pursuit-evasion games, cops and robbers, cop-win graphs, capture time, extremal graphs.

2010 Mathematics Subject Classification: 05C57, 05C75, 05C35.

\section{REFERENCES}

[1] A. Bonato and R. Nowakowski, The Game of Cops and Robbers on Graphs (AMS Student Mathematical Library, 2011). 
[2] A. Bonato, P. Golovach G. Hahn and J. Kratochvil, The capture time of a graph, Discrete Math. 309 (2009) 5588-5595. https://doi.org/10.1016/j.disc.2008.04.004

[3] N. Clarke, S. Finbow and G. MacGillivray, A simple method of computing the catch time, Ars Math. Contemp. 7 (2014) 353-359. https://doi.org/10.26493/1855-3974.304.fec

[4] T. Gavenciak, Cop-win graphs with maximum capture-time, Discrete Math. 310 (2010) 1557-1563. https://doi.org/10.1016/j.disc.2010.01.015

[5] W. Kinnersley, Bounds on the length of a game of Cops and Robbers, Discrete Math. 341 (2018) 2508-2518. https://doi.org/10.1016/j.disc.2018.05.025

[6] R. Nowakowski and P. Winkler, Vertex-to-vertex pursuit in a graph, Discrete Math. 43 (1983) 235-239. https://doi.org/10.1016/0012-365X(83)90160-7

[7] D. Offner and K. Ojakian, Cop-win graphs: Optimal strategies and corner rank (2017).

arXiv: org/abs/1607.03471

[8] D. Offner and K. Ojakian, Graphical examples of cop-win graphs. arXiv: org/abs/1703.04427

[9] A. Quilliot, Jeux et Pointes Fixes sur les Graphes (Ph.D. Dissertation, Université de Paris VI, 1978).

Received 27 November 2017

Revised 19 March 2019

Accepted 19 March 2019

\section{APPENDIX}

The results proved in this appendix are included to keep the paper self-contained. However more general results, that include those here, are contained in our submitted paper [7] which was unpublished at the time this paper was published. Similar results are proved in [3]; the relationship to our approach is discussed in [7].

The proof that the corner ranking procedure is well defined follows.

Proof. To show that the corner ranking procedure is well-defined on cop-win graphs, it suffices to show if $G$ is a cop-win graph which is not a clique, then $G$ must have a strict corner. Supposing $G$ is a non-clique which has no strict corners, we show that it is not cop-win. Let $v \in V(G)$ be some corner, and let $v_{1}, \ldots, v_{k}$ be all the vertices which corner $v$, which means that any two vertices 
among $v, v_{1}, \ldots, v_{k}$ are twins. Using the idea of corner elimination, $G$ is cop-win if and only if the graph $G^{\prime}$ obtained by deleting the corners $v_{1}, \ldots, v_{k}$ is cop-win. Note that $v$ is not a corner in $G^{\prime}$, and since $G$ was not a clique, $G^{\prime}$ is not a clique. If $G^{\prime}$ has no corners, then $G$ is not cop-win. Otherwise, repeating the removal process for the remaining sets of twins in $G^{\prime}$ will eventually result in a graph that has no corners. Thus $G$ is not cop-win.

The proof of Lemma ?? follows.

Proof. Suppose $\left(v_{1}, \ldots, v_{k}\right)$ is a maximal sequence of strict corners such that $v_{1}=v$ and each $v_{i+1}$ strictly corners $v_{i}$. Note that $v_{j}$ strictly corners $v_{i}$ if $i<j$, so $v_{k}$ strictly corners $v$. Since $v_{k}$ is a strict corner, it must be strictly cornered by some vertex $w \notin\left\{v_{1}, \ldots, v_{k}\right\}$. By the maximality of the sequence, $w$ is not a strict corner. Since $v$ is strictly cornered by $v_{k}$ and $v_{k}$ is strictly cornered by $w$, $v$ is strictly cornered by $w$, which is not a strict corner, and thus $w$ is of higher rank.

To prove Theorem ??, we need to define projection functions relative to corner rank. For any graph $G$, define $\mathcal{P}(G)$ to be the non-empty subsets of $V(G)$.

Definition. Suppose $G$ is a graph with corner rank $\alpha$. We define the functions $f_{1}, \ldots, f_{\alpha-1}$ and $F_{1}, \ldots, F_{\alpha-1}, F_{\alpha}$, where $f_{k}: \mathcal{P}\left(G^{[k]}\right) \rightarrow \mathcal{P}\left(G^{[k+1]}\right)$ and $F_{k}:$ $\mathcal{P}(G) \rightarrow \mathcal{P}\left(G^{[k]}\right)$.

- For a single vertex $u \in V\left(G^{[k]}\right)$, define

$$
\begin{aligned}
& f_{k}(\{u\})= \begin{cases}\{u\} & \text { if } \operatorname{cr}(u)>k \\
\text { the set of vertices in } G^{[k+1]} \text { that strictly corner } u \text { in } G^{[k]} & \text { if } \operatorname{cr}(u)=k .\end{cases} \\
- & f_{k}\left(\left\{u_{1}, \ldots, u_{t}\right\}\right)=\bigcup_{1 \leq i \leq t} f_{k}\left(\left\{u_{i}\right\}\right) . \\
\text { - Let } F_{1}: \mathcal{P}(G) \rightarrow \mathcal{P}(G) \text { be the identity function. } & \text { - For } k \geq 2, \text { let } F_{k}=f_{k-1} \circ \cdots \circ f_{1} .
\end{aligned}
$$

For a function $h$ whose domain is sets of vertices, we adopt the usual convention that $h(u)=h(\{u\})$ for a single vertex $u$. We remark that by by Lemma ?? the functions $f_{k}$ are guaranteed to have non-empty sets for values. We say $v$ is a $k$-projection (or simply a projection) of $w$ if $v \in F_{k}(w)$.

Definition. Let $H$ and $G$ be two graphs. We say the function $h: \mathcal{P}(H) \rightarrow \mathcal{P}(G)$ is a homomorphism if all the vertices of $h(U)$ are adjacent to all the vertices of $h(V)$ whenever all the vertices of $U$ are adjacent to all the vertices of $V$.

Lemma. For any graph with corner rank $\alpha$, its associated functions $f_{1}, \ldots, f_{\alpha-1}$ and $F_{1}, \ldots, F_{\alpha-1}, F_{\alpha}$ are homomorphisms. 
Proof. Let $G$ be the graph. The identity function $F_{1}$ is a homomorphism. We show that each $f_{k}$ is a homomorphism, which implies all other $F_{k}$ 's are homomorphisms because the property is preserved by composition. We prove that $f_{k}$ is a homomorphism when the sets $U$ and $V$ consist of just the single vertices $u$ and $v$, respectively. The general case then follows immediately. Suppose $u, v \in V\left(G^{[k]}\right)$ are distinct and adjacent, and let $u^{*} \in f_{k}(u)$ and $v^{*} \in f_{k}(v)$; we show that $u^{*}$ and $v^{*}$ are adjacent. Note that even if $u^{*}=v^{*}$, the argument works since our graphs are reflexive.

Case 1. Suppose $u, v \in V\left(G^{[k+1]}\right)$. Then $f_{k}(v)=\{v\}$ and $f_{k}(u)=\{u\}$, and so $u^{*}=u$ and $v^{*}=v$ are adjacent.

Case 2. Suppose $v \in V\left(G^{[k+1]}\right), u \notin V\left(G^{[k+1]}\right)$. So $v^{*}=v$ and $u^{*} \neq u$. Since $u^{*}$ strictly corners $u$ in $G^{[k]}, u^{*}$ is adjacent to $v$, and thus $u^{*}$ and $v^{*}$ are adjacent.

Case 3. Suppose $u, v \notin V\left(G^{[k+1]}\right)$. So $v^{*} \neq v$ and $u^{*} \neq u$. Since $u^{*}$ strictly corners $u$ in $G^{[k]}, u^{*}$ is adjacent to both $u$ and $v$. Since $v^{*}$ strictly corners $v$ in $G^{[k]}$, and $v$ is adjacent to $u^{*}$, we have that $v^{*}$ is also adjacent to $u^{*}$.

The proof of Theorem ?? follows.

Proof. Let $G$ be a $t$-top cop-win graph with corner rank $\alpha$. To show that $\operatorname{capt}(G)=\alpha-t$, we prove an upper and lower bound on $\operatorname{capt}(G)$.

First we show capt $(G) \leq \alpha-t$. For $k \geq 1$, we say that the robber is $k$-caught if the cop is at some vertex $c$, the robber at some vertex $r$, and $c \in F_{k}(r)$. We describe a strategy on $G$ for the cop that succeeds in at most $\alpha-t$ cop moves. The cop starts at any vertex of corner rank $\alpha$. No matter where the robber starts, if $G$ is 1-top, then since the cop dominates the top two ranks of vertices, the cop can play so after one cop move, the robber is $(\alpha-1)$-caught. Similarly, if $G$ is 0 -top, then the cop can play so that after one move the robber is $\alpha$-caught. We show the following claim:

If the robber is $k$-caught, for $k \geq 2$, then for any robber move, there is a cop move which leaves the robber $(k-1)$-caught.

Proving the claim proves the upper bound since we can repeatedly apply the claim and once the robber is 1-caught, the robber is actually caught. Now we prove the claim, where we suppose the cop is at $c$ and the robber is at $r$. Since $c \in F_{k}(r)=f_{k-1} \circ F_{k-1}(r)$, either $c \in F_{k-1}(r)$ or there is an $r^{\prime} \in F_{k-1}(r)$ such that $c$ strictly corners $r^{\prime}$ in $G^{[k-1]}$. Either way, there is a $(k-1)$-projection $r^{\prime}$ of $r$ such that $c$ corners $r^{\prime}$ in $G^{[k-1]}$. Thus, since $F_{k-1}$ is a homomorphism, wherever the robber moves to, from $r$, the cop can move so that the robber is $(k-1)$-caught. 
Now we show $\operatorname{capt}(G) \geq \alpha-t$. We say that a robber location at vertex $r$ is $k$-proj-safe if its corner rank is at least $k$ and the cop is at a vertex $c$ such that there is some $c^{\prime} \in F_{k}(c)$ such that $c^{\prime}$ is not adjacent to $r$. Since $F_{k}$ is a homomorphism, if a location is $k$-proj-safe, then the cop is not adjacent to the robber.

If $G$ is 0 -top, then the robber starts at a vertex which is $(\alpha-1)$-proj-safe, while if $G$ is 1-top, then the robber starts at a vertex which is $(\alpha-2)$-proj-safe. We show that starting in such a way is possible. We use the fact that since $F_{k}$ is a homomorphism, the vertices of rank $k$ or higher adjacent to a vertex $c$ are a subset of the vertices of rank $k$ or higher adjacent to a vertex from $F_{k}(c)$. Thus to see that a $(\alpha-1)$-proj-safe start is possible in the 0 -top case, it suffices to show there is no vertex in $G^{[\alpha-1]}$ that dominates $G^{[\alpha-1]}$. If there were such a vertex, it would have to be rank $\alpha$, but then the graph would be 1-top. Similarly, to see that such a start is possible in the 1-top case, it suffices to show that there is no vertex in $G^{[\alpha-2]}$ that dominates $G^{[\alpha-2]}$. Suppose for the sake of contradiction there is such a vertex $v$. In $G^{[\alpha-2]}, v$ cannot strictly corner any vertex in $G^{[\alpha-1]}$, so all the vertices of $G^{[\alpha-1]}$ are twins with $v$. But the fact that a vertex of rank $\alpha-1$ is twins with a vertex of rank $\alpha$ leads to a contradiction. The lower bound will follow once we prove the following claim:

If the robber location is $k$-proj-safe, for $k \geq 2$, then no matter what the cop does, the robber has a move to a $(k-1)$-proj-safe location.

To prove the claim, suppose the robber is at the $k$-proj-safe vertex $r_{0}$, and the cop is at $c_{0}$. Thus there exists $c_{0}^{\prime} \in F_{k}\left(c_{0}\right)$ such that $c_{0}^{\prime}$ is not adjacent to $r_{0}$. Suppose the cop then moves to $c_{1}$. Assume for the sake of contradiction that from $r_{0}$ the robber does not have a move to a $(k-1)$-proj-safe vertex. For $r_{0}$ not to have such a move, means that all $c_{1}^{\prime} \in F_{k-1}\left(c_{1}\right)$ corner $r_{0}$ in $G^{[k-1]}$. Consider one such $c_{1}^{\prime}$. Since $r_{0}$ has rank at least $k$, and thus cannot be a strict corner in $G^{[k-1]}$, this cornering cannot be strict, and thus $c_{1}^{\prime}$ and $r_{0}$ are twins in $G^{[k-1]}$. Since $r_{0}$ has rank at least $k, c_{1}^{\prime}$ must also have rank at least $k$. This implies that $f_{k-1}\left(c_{1}^{\prime}\right)=\left\{c_{1}^{\prime}\right\}$ and so $c_{1}^{\prime} \in F_{k}\left(c_{1}\right)=f_{k-1} \circ F_{k-1}\left(c_{1}\right)$. However since $F_{k}$ is a homomorphism and $c_{1}$ is adjacent to $c_{0}, c_{1}^{\prime}$ is adjacent to $c_{0}^{\prime}$. Since $r_{0}$ is not adjacent to $c_{0}^{\prime}$, this contradicts the fact that $c_{1}^{\prime}$ and $r_{0}$ are twins in $G^{[k-1]}$. 